\title{
Mountain tea (Sideritis plants): A potential anti-atherogenic agent?
}

\author{
Ekaterina-Michaela Tomou ${ }^{1,2}$, Despina N. Perrea ${ }^{2}$, Helen Skaltsa ${ }^{1}$ \\ 'Laboratory of Pharmacognosy and Chemistry of Natural Products, School of Pharmacy, National \& Kapodistrian \\ University of Athens, Panepistimiopolis, Zografou, Athens, Greece \\ 'Laboratory for Experimental Surgery and Surgical Research "N.S Christeas", Athens Medical School, National \\ \& Kapodistrian University of Athens, Athens, Greece
}

\begin{abstract}
Atherosclerosis consists the leading cause of cardiovascular diseases. Over the last years, medicinal plants are considered as efficient agents in the prevention and adjuvant therapy of atherosclerosis. Mountain tea (Sideritis plants) is used as traditional remedy against common cold and gastrointestinal disorders. Its beneficial properties are attributed to its rich bioactive constituents. Polyphenols exhibit a broad range of pharmacological activities such as antioxidant, anti-inflammatory and anti-atherosclerotic effects. The present review summarizes and discusses the potential of mountain tea as an anti-atherogenic agent based on its bioactive chemical compounds and their reported pharmacological activities.
\end{abstract}

KEY WORDS: Mountain tea, genus Sideritis, antioxidant, anti-inflammatory, potential anti-atherogenic activity, polyphenols

\section{INTRODUCTION}

Cardiovascular diseases (CVD) consist the number one cause of death worldwide, representing the $31 \%$ of all the deaths in the world ${ }^{1}$. Major role in the development of these diseases holds atherosclerosis ${ }^{2}$, a multifactional chronic disease associated with inflammation, oxidative stress, endothelial dysfunction, and aging ${ }^{3,4}$. Furthermore, external risk factors such as smoking, obesity and unhealthy diet could contribute to the development of

\section{Corresponding author:}

Prof. H. Skaltsa

Department of Pharmacognosy and Chemistry of Natural Products, School of Pharmacy, National \& Kapodistrian University of Athens

Panepistimiopolis, Zografou, 15771, Athens, Greece

Tel./Fax.: +30 2107274593

E-mail: skaltsa@pharm.uoa.gr atherosclerosis ${ }^{2}$. To prevent its progression in any stage, the adoption of healthy lifestyle interventions from early age seems to be great essential. Healthy diet, physical activity and abstinence from tobacco are basic recommendations of clinical strategies for its prevention ${ }^{2}$.

Medicinal plants are predominant ingredients of healthy diets and especially of Mediterranean diet. A current review carried out by Kirichenko et al. (2020) thoroughly described the medicinal plants which have exerted anti-atherosclerotic activity in experimental and clinical studies ${ }^{5}$. This activity is mainly attributed to their content of bioactive compounds with pleiotropic effects such as antioxidant and anti-inflammatory properties, acting with different mechanisms of actions $s^{5,6}$. Medicinal plants with potential anti-atherosclerotic activity are especially interesting, since generally natural products

Submission: 04.03.2021, Acceptance: 21.04.2021 
are considered as safer agents compared to conventional drugs due to their fewer side effects and are suitable for long-term use. Therefore, plant-based medications could be used as suitable candidates for a long-term application for prevention and adjuvant treatment of atherosclerosis.

Plants of Lamiaceae family are well-known medicinal plants with a broad range of pharmacological activities. Many of them are extensively used in traditional medicine for various applications since antiquity. In recent years, a genus of this family which has attracted great scientific interest is genus Sideritis L. Its traditional beverages, widely well-known as mountain tea, have been the subject of various phytochemical and pharmacological studies due to their significant health benefits. Of great importance are its antioxidant and anti-inflammatory activities, which have been thoroughly investigated by in vitro and in vivo studies. However, so far, there is no report on its anti-atherogenic effects. Thus, the aim of the present review was to summarize and discuss the potential of this important genus as an anti-atherogenic agent based on its bioactive chemical compounds and their reported pharmacological activities.

\section{Methods}

A comprehensive research of previously published literature data about genus Sideritis was performed. Electronic databases including Scopus, PubMed, and Google Scholar were searched with keywords related to the antiatherosclerotic activity of genus Sideritis, its bioactive compounds (e.g. flavonoids, phenylethanoid glycosides) and their pharmacological activity.

\section{Bioactive compounds of Mountain Tea}

Phytochemical studies in genus Sideritis have revealed the presence of many phytochemicals, mainly polyphenols including flavonoids, phenylethanoid glycosides and phenolic acids ${ }^{7-9}$. Regarding the flavonoid load, Sideritis spp. are characterized by 8-hydroxyflavone 7-allosylglucosides (isoscutellarein and hypolaetin derivatives). In addition, these plants contain 5,7-dihydroxyflavones (apigenin derivatives) and their glucosides. Another common chemical category of genus Sideritis is the phenylethanoid glycosides. Acteoside and martynoside were identified from several Sideritis plants. Although plants of Lamiaceae family are rich in diverse phenolic acids, chlorogenic acid is the major representative in Sideritis species. It is noteworthy to point out that the variations of the phytochemical content among Sideritis plants depend on various factors such as environmental conditions and geographical origin of plant materials.

\section{Flavonoids}

Previous studies have showed that the consumption of flavonoid-rich diets can reduce the risk of cardiovascular diseases and atherosclerosis ${ }^{10,11}$. Particularly, flavonoids can act with various mechanisms of action in atherosclerotic progression, including antioxidant, anti-inflammatory, antiplatelet, vasodilatory, antihypertensive and lipid regulation ${ }^{10,12}$. Concerning the antioxidant activity, they mainly act through direct scavenging of free radicals, metal chelation, inhibition of ROS producing enzymes (e.g. lipoxygenases, NADPH oxidases), up-regulating of cellular antioxidants, inhibition of LDL oxidation, induction of antioxidant enzymes and inhibition of NF-kB pathway. Furthermore, flavonoids exhibit anti-inflammatory activity since they decrease enzymes which participate on inflammation pathways such as cyclooxygenases (COX-1 and mainly COX-2) and lipoxygenases, as well as they reduce NO production. In addition, these constituents are capable of diminishing the expression of pro-inflammatory cytokines (e.g. IL-1 b, IL-6, IL-8, TNFa) and regulate NF-KB activation. Some flavonoids also possess anti-platelet effects, acting as reverse antagonisms on the thromboxane $\mathrm{A} 2$ receptor and inhibiting serotonin, collagen, ADP induced platelet coagulation, PAF, $P$-selectin and calcium mobilization. Though, they induce PECAM-1 activation. Additionally, flavonoids increase validation and enhance the endothelial function through improving eNOS activity and expression, the prostacyclin production, and the increase of EDHF-mediated relaxation, whereas they inhibit iNOS and ET-1 action and synthesis. Lipid accumulation which is observed in atherosclerosis is also regulated by flavonoids through decreasing cholesterol synthesis, hepatic secretion of ApoB-100 and foam cell formation, as well as, they upregulate LDL, HDL, fatty acid metabolizing enzymes and PPARY.

Flavonoids are main constituents of Sideritis species. Among them, kaempferol (3-hydroxyflavone) and apigenin (flavone) have been extensively investigated for their great bioactivities, including antioxidant, anti-inflammatory and cardioprotective effects ${ }^{10,11,13,14}$, revealing them as potential preventative anti-atherogenic agents. Characteristic flavonoids of Sideritis species are glycosides, acetylated or not, of isoscutellarein and hypolaetin, as well as their methylated derivatives. The antioxidant and anti-inflammatory effects of these constituents have been reported previously ${ }^{7,15}$. Consequently, it is expected that mountain tea could also exert anti-atherogenic activity due to its bioactive flavonoids.

\section{Phenylethanoid glycosides}

Phenylethanoid glycosides are phenolic derivatives which characterize genus Sideritis. Numerous pharmacolog- 
ical activities have been reported such as antioxidant, antiinflammatory, and anti-hypertensive effects ${ }^{16}$. Regarding their antioxidant properties, major mechanisms of action are through the direct scavenging of free radicals, metal chelation, and inhibition of ROS producing enzymes ${ }^{17}$. Moreover, these compounds have shown anti-inflammatory activity, resulting in the reduction of cyclooxygenases, lipoxygenases, NO production, pro-inflammatory cytokines and regulation of NF-KB activation ${ }^{17}$. Acteoside is one of the most occurrent phenylethanoid glycoside in Sideritis species with a broad range of pharmacological activities, mainly antioxidant and anti-inflammatory ${ }^{18}$. Martin-Nizard et al., (2003) exhibited that four phenylethanoid glycosides, including acteoside, inhibited LDL oxidation in vitro ${ }^{19}$. Furthermore, these compounds were able to completely abolish the capacity of copper-oxidized LDL (Cu-LDL)-induced BAEC ET-1 liberation ${ }^{20}$, and revealed anti-atherosclerotic effects, since ET-1 secretion is increased in atheroma and promote atherosclerosis. Another study carried out by Chiou et al. (2003) mentioned that acteoside reduced the risk of atherosclerosis, not only by protecting LDL from oxidative modification, but also by its free radical-scavenging properties ${ }^{21}$. In addition, acteoside demonstrated inhibition of cell adhesion molecules (CAMs) which are involved in the pathogenesis of atherosclerosis and inflammation by decreasing phosphorylation of extracellular signalregulated kinase (ERK) and c-Jun N-terminal kinase (JNK) ${ }^{22}$. Currently, acteoside showed to reduce the expression of inflammatory mediators (NO, COX-2 and prostaglandin E2) and to suppress the phosphorylation of NF-KB in primary rat chondrocytes treated with $\mathrm{IL}-1 \mathrm{~b}^{23}$. Notably, acteoside at high dosage through oral administration does not cause genotoxicity ${ }^{23}$. The above findings indicate that acteoside could be considered as a promising natural agent to attenuate or prevent the development of atherosclerosis. This bioactive compound which is a major constituent in mountain tea could contribute to its potential antiatherogenic effects.

\section{Phenolic acids}

Phenolic acids are widely well-known for their im- portant biological activities ${ }^{24}$. Chlorogenic acid is one of the most abundant phenolic acids in human diet and in species of genus Sideritis. In recent years, chlorogenic acid has gained considerable attention due to its great antioxidant, anti-inflammatory, anti-diabetic, anti-obesity, and antihypertension properties ${ }^{25}$. It is considered as a potential natural anti-atherosclerotic agent because of its hypolipidemic, anti-inflammatory, antioxidative, antiplatelet and vascular endothelial properties ${ }^{26}$. Wu et al. (2014) evaluated the effect of chlorogenic acid on atherosclerosis development in vivo in ApoE knock-out mice, as well as its mechanism of action ${ }^{27}$. They mentioned that this phenolic compound decreased the atherosclerotic lesion area and vascular dilatation in aortic root compared to the control compound, atorvastatin. Furthermore, chlorogenic acid reduced cholesterol, triglycerides, LDL and inflammatory markers in plasma. The oxLDL-induced lipid accumulation was suppressed by chlorogenic derivatives in RAW264.7 cells, as well as cholesterol efflux was stimulated. In addition, chlorogenic acid improved the mRNA levels of PPARY, LXRa, ABCA1, ABCG1 and the transcriptional activity of PPARY.

\section{CONCLUSION}

The data presented here demonstrate that mountain tea potentially reduces atherosclerosis development due to its rich content in polyphenols. Polyphenols have been thoroughly studied for their significant pharmacological effects. Of great importance are their antioxidant and anti-inflammatory activities. Thus, mountain tea could exert potent anti-atherogenic effects, in parallel to its strong antioxidant and anti-inflammatory capacity. It can be suggested that it could be used as a natural agent in terms of prevention and treatment of atherosclerosis. However, further in vivo studies are necessary in order to specify its anti-atherogenic effects and its exact mechanisms of action.

\section{Funding}

The present study was supported by a research grant from the Hellenic Atherosclerosis Society. 


\title{
ПЕРІАНЧH
}

\section{Toál tou ßouvoú: ПıӨavóç avtl-aOnpoyóvoc mapáyovtac;}

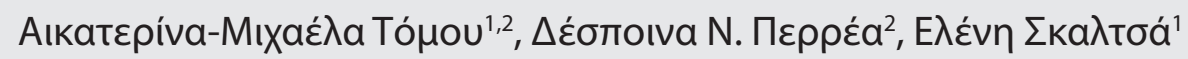

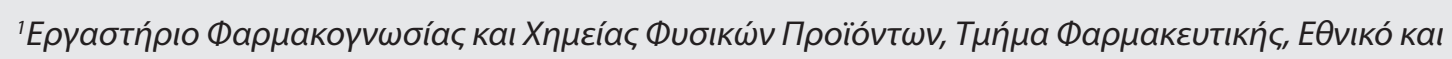

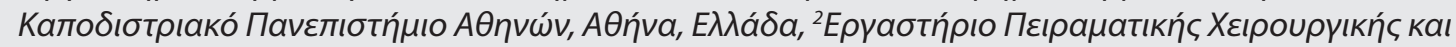

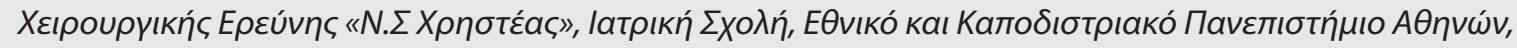

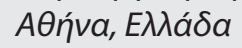

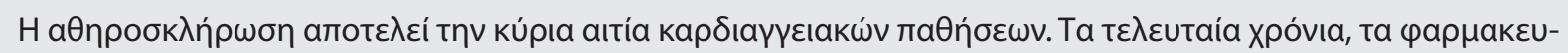

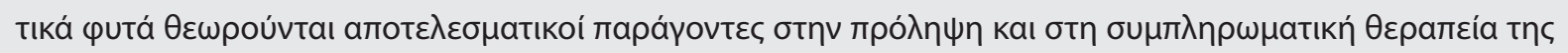

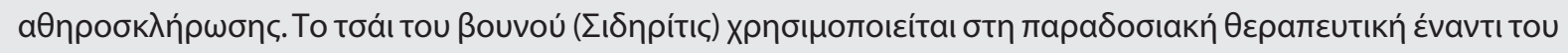

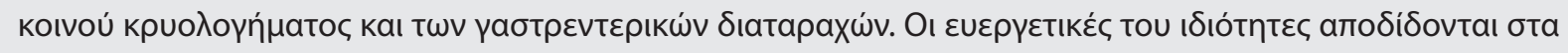

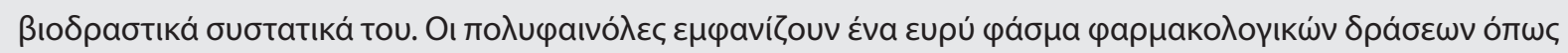

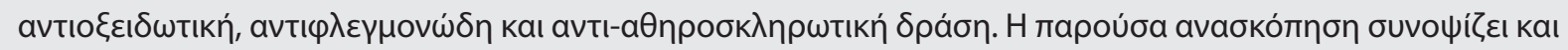

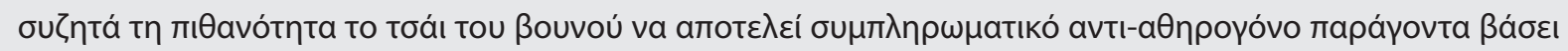

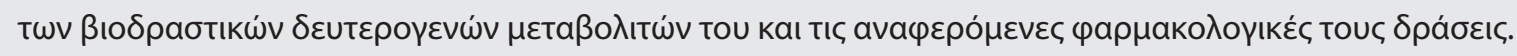

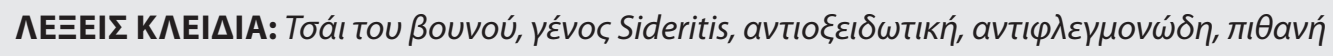

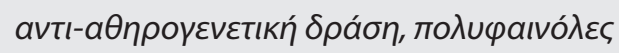

\section{REFERENCES}

1. World Health Organisation (WHO). www.who.int (Accessed on 12 April 2021).

2. Libby P, Buring JE, Badimon L, Hansson GK, Deanfield J, Bittencourt MS, et al. Atherosclerosis. Nat Rev Dis Primers. 2019 Aug;5(1):56.

3. Shemiakova T, Ivanova E, Grechko AV, Gerasimova EV, Sobenin IA, Orekhov AN. Mitochondrial dysfunction and DNA damage in the context of pathogenesis of atherosclerosis. Biomedicines. 2020 Jun;8(6):166.

4. Marchio P, Guerra-Ojeda S, Vila JM, Aldasoro M, Victor VM, Mauricio MD. Targeting early atherosclerosis: A focus on oxidative stress and inflammation. Oxid. Med. Cell. Longev. 2019 Jul;2019:1-32.

5. Kirichenko TV, Sukhorukov VN, Markin AM, Nikiforov NG, Liu PY, Sobenin IA, et al. Medicinal plants as a potential and successful treatment option in the context of atherosclerosis. Front Pharmacol. 2020 Apr; 11:403.

6. Sharif H, Akash MSH, Rehman K, Irshad K, Imran I. Pathophysiology of atherosclerosis: Association of risk factors and treatment strategies using plant-based bioactive compounds. J Food Biochem. 2020 Nov;44:e13449. Available from: https://onlinelibrary.wiley.com/doi/ abs/10.1111/jfbc.13449/

7. González-Burgos E, Carretero ME, Gómez-Serranillos MP. Sideritis spp.: Uses, chemical composition and pharmacological activities-a review. J Ethnopharmacol. 2011 May;135(2):209-25.

8. Aneva I, Zhelev P, Kozuharova E, Danova K, Nabavi SF, Behzad, S. Genus Sideritis, section Empedoclia in southeastern Europe and Turkey- studies in ethnopharmacology and recent progress of biological activities. DARU J
Pharm Sci. 2019 Jun;27(1):407-21.

9. Żyżelewicz D, Kulbat-Warycha K, Oracz J, Żyżelewicz K. Polyphenols and other bioactive compounds of sideritis plants and their potential biological activity. Molecules. 2020 Aug;25(16):3763.

10. Ciumărnean L, Milaciu MV, Runcan $O$, Vesa ȘC, Răchișan $A L$, Negrean V, et al. The effects of flavonoids in cardiovascular diseases. Molecules. 2020 Sep;25(18):4320.

11. Siasos G, Tousoulis D, Tsigkou V, Kokkou E. Oikonomou E, Vavuranakis M, et al. Flavonoids in atherosclerosis: An overview of their mechanisms of action. Curr Med Chem. 2013 April;20(21):2641-60.

12. Grassi D, Desideri G, Ferri C. Flavonoids: Antioxidants against atherosclerosis. Nutrients. 2010 Aug; 2(8): 889-902.

13. Salvamani S, Gunasekaran B, Shaharuddin NA, Ahmad SA, Shukor MY. Antiartherosclerotic effects of plant flavonoids. Biomed Res Int. 2014 May;2014:480258. Available from: https://pubmed.ncbi.nlm.nih.gov/24971331/

14. Alam W, Khan H, Shah MA, Cauli O, Saso L. Kaempferol as a dietary anti-inflammatory agent: Current therapeutic standing. Molecules. 2020 Sep;25(18):4073.

15. Güvenç A, Okada Y, Akkol EK, Duman H, Okuyama T, Çalış i. Investigations of anti-inflammatory, antinociceptive, antioxidant and aldose reductase inhibitory activities of phenolic compounds from Sideritis brevibracteata. Food Chem. 2010 Feb;118(3):686-92.

16. Tian X.-Y, Li M-X, Lin T, Qiu Y, Zhu Y-T, Li X-L, et al. A review on the structure and pharmacological activity of phenylethanoid glycosides. Eur J Med Chem. 2020 Jan; 209:112563. Available from: https://pubmed.ncbi.nlm. nih.gov/33038797/

17. Xue Z, Yang B. Phenylethanoid glycosides: Research ad- 
vances in their phytochemistry, pharmacological activity and pharmacokinetics. Molecules. 2016 Jul;21 (8):991.

18. He J, Hu XP, Zeng Y, Li Y, Wu HQ, Qiu RZ, et al. Advanced research on acteoside for chemistry and bioactivities. J Asian Nat Prod Res. 2011 May;13(5):449-64.

19. Martin-Nizard F, Sahpaz S, Furman C, Fruchart JC, Duriez P, Bailleul F. Natural phenylpropanoids protect endothelial cells against oxidized LDL-induced cytotoxicity. Planta Med. 2003 Mar;69(3):207-11.

20. Martin-Nizard F, Sahpaz S, Kandoussi A, Carpentier M, Fruchart JC, Duriez P, et al. Natural phenylpropanoids inhibit lipoprotein-induced endothelin-1 secretion by endothelial cells. J Pharm Pharmacol. 2004 Dec;56(12):160711.

21. Chiou W-F, Lin L-C, Chen C-F. The antioxidant and free radical scavenging properties of acteoside. Chin Pharm J. (Taipei) 2003 Oct;55:347-53.

22. Chen C-H, Song T-Y, Liang Y-C, Hu M.-L. Acteoside and 6-O-Acetylacteoside Downregulate Cell Adhesion Molecules Induced by IL-1 $\beta$ through Inhibition of ERK and JNK in human vascular endothelial cells. J Agric Food Chem. 2009 Oct;57(19): 8852-9.

23. Lim H, Kim DK, Kim TH, Kang KR, Seo JY, Cho SS, et al. Acteoside counteracts interleukin-1 $\beta$-induced catabolic processes through the modulation of mitogen-activated protein kinases and the NFKB cellular signaling pathway. Oxid Med Cell Longev. 2021 Mar;2021:8684725. Available from: https://pubmed.ncbi.nlm.nih.gov/33833854/

24. Kumar N, Goel N. Phenolic acids: Natural versatile molecules with promising therapeutic applications. Biotechnol Rep (Amst). 2019 Aug;24:e00370. Available from: https:// pubmed.ncbi.nlm.nih.gov/31516850/

25. Naveed M, Hejazi V, Abbas M, Kamboh AA, Khan GJ, Shumzaid $M$, et al. Chlorogenic acid (CGA): A pharmacological review and call for further research. Biomed Pharmacother. 2018 Jan;97:67-74. Available from: https:// pubmed.ncbi.nlm.nih.gov/29080460/

26. Li L, Su C, Chen X, Wang Q, Jiao W, Luo H, et al. Chlorogenic acids in cardiovascular disease: A review of dietary consumption, pharmacology, and pharmacokinetics. J. Agric. Food Chem. 2020 Jun; 68, 24, 6464-84. Available from: https://pubmed.ncbi.nlm.nih.gov/32441927/

27. Wu C, Luan H, Zhang X, Wang S, Zhang X, Sun X, Guo P. Chlorogenic acid protects against atherosclerosis in ApoE-/- Mice and promotes cholesterol efflux from RAW264.7 Macrophages. PLoS ONE 2014 Sep; 9(9):e95452. Available from: https://www.ncbi.nlm.nih.gov/pmc/ articles/PMC4154672/ 